\title{
Streamline Simulation and Analysis of Pedestrian Weaving Flow in Large Passenger Terminal
}

\author{
Jun Li, ${ }^{1,2}$ Jing Wang, ${ }^{2}$ Yuanfang Dong, ${ }^{3}$ Hongfei Jia, ${ }^{1}$ and Yanzhong $\mathrm{Li}^{2,4}$ \\ ${ }^{1}$ College of Traffic, Jilin University, Changchun, Jilin 130025, China \\ ${ }^{2}$ Department of Applied Mathematics, Changchun University of Science and Technology, Changchun, Jilin 130022, China \\ ${ }^{3}$ School of Economics and Management, Changchun University of Science and Technology, Changchun, Jilin 130022, China \\ ${ }^{4}$ College of Mathematics and Statistics, Beihua University, Jilin 132013, China
}

Correspondence should be addressed to Hongfei Jia; jiahf@jlu.edu.cn

Received 10 June 2014; Revised 23 November 2014; Accepted 26 November 2014

Academic Editor: Efstratios Tzirtzilakis

Copyright (C) 2015 Jun Li et al. This is an open access article distributed under the Creative Commons Attribution License, which permits unrestricted use, distribution, and reproduction in any medium, provided the original work is properly cited.

\begin{abstract}
A new concept called the extended weaving area is proposed to relieve the conflicts and clogging caused by pedestrian weaving in both time and space in large passenger terminal. The cellular automaton model that considers pedestrian walking habits based on the floor field is adopted. Numerical simulations are carried out in MATLAB environment to explore the relationship between the emptying time and bottleneck setting when four groups of pedestrians walk to four exits through the weaving areas with different settings. It is found that, by using improved extended weaving area settings, the stress of the weaving area could be relieved in both time and space; thus the efficiency of pedestrians passing could be improved. Based on the simulation, the threshold of single bottleneck width in the extended weaving area is also given in this research.
\end{abstract}

\section{Introduction}

The complex behavior conducted by pedestrian flow includes jam transition, clogging, lane formation, and "faster is slower." The meaning of "faster is slower" is pedestrians trying to move faster but cause a smaller average speed of leaving. These phenomena can be investigated from the perspective of physics [1]. Therefore, many physical models, such as cellular automaton (CA) models [2-15], social force models [16-18], and fluid dynamic models [19], have been applied to research pedestrian crowd dynamics and have achieved great success in the community. Some investigations have explored the fundamental diagrams between density and flow or density and velocity $[7,14,20-22]$. These fundamental diagrams can be used to evaluate models of pedestrian flow.

In the transportation research field, cellular automaton is becoming more and more significant and has been the most common choice, for it can not only model individual behaviors but also reflect the characteristics of collective dynamics. Characteristics of collective dynamics include jamming, lane formation, oscillation, patterns at intersection, trail formation, panics $[15,16]$. The CA applications on characteristic analysis of pedestrian flow include bidirectional flow $[2,3]$, fire evacuation [4], evacuation under poor visibility [5], airplane evacuation [6], T crossing [7], evacuation with obstacles $[8,9]$, evacuation in ramp $[10]$, and cooperative and competitive behaviors [6].

Most of the CA models discretize the floor into rectangular cells and give a weight to each cell in every step of the iteration. The calculation of weight depends on the location and the width of the exits, pedestrians' emotion, and location of obstacles. Burstedde et al. [11] first proposed the idea of floor field to compute the weights and simulated the pedestrian dynamic in counter flow.

There are two kinds of floor field, namely, the static floor field and the dynamic floor field. The static floor field does not change with time nor does it change with appearance of pedestrians. Huang and Guo [12] discussed the choice of exits in multiexits evacuation under the circumstance with obstacles and also suggested an iterative calculating method of the static floor field.

The dynamic floor field, however, should change with appearance of pedestrians. It should adjust the weight in every step, and the parameters considered include pedestrian 
behavior, pedestrian distribution, pedestrian density near exit, and the distance between the pedestrian and the exit. The pedestrian movement depends on the local motion rules and the weight of each cell.

Concerning streamline weaving of pedestrian flow, Chen et al. [13] proposed a lattice gas model based on a floor field and studied the characteristics and clogging mechanism of pedestrian weaving with two various directions. Yao et al. [14] classified the phenomena of pedestrian weaving flow into three parts: forward weaving behavior, cross weaving behavior, and lateral weaving behavior. Several quantization parameters are given, and correlation between different behavioral parameters is analyzed based on real data of DongZhiMen hub [14].

Weaving area in traffic highway is also studied by using cellular automaton [23-25]. Nishi et al. [23] have studied the effect of vehicular configuration on the weaving traffic flow on a two-lane highway by using cellular automaton model. Li et al. [24] investigated capacity of the weaving section by using cellular automaton; they argued that the length of the weaving section should be larger than $150 \mathrm{~m}$. Lei et al. [25] analyzed the weaving section with one-lane main road based on the NS cellular automaton traffic model, and the simulation results suggested that a proper length of weaving sections needs to be chosen.

In this paper, we model pedestrian weaving flow in a large passenger terminal modeled as a rectangle grid space with two channels and set an extended weaving area between the channels. In this way, pedestrians in the four directions form the weaving streamlines in the weaving area. We utilize CA model based on the floor field and pedestrian walking habits to study the characteristics of pedestrian weaving flow and give the threshold of bottleneck's width based on simulated comparative studies.

The rest of this paper is organized as follows. Section 2 introduces the setting mode of the weaving area. Section 3 describes the cellular automaton model based on walking habits and the floor field. The experiments of numerical simulation and the analysis are given in Section 4. Section 5 draws the conclusions.

\section{Setting Mode of the Weaving Area}

In large passenger terminal and traffic terminal, a phenomenon often occurs where pedestrians come across each other in the weaving area while they are in different directions. The administration often tries to avoid jam and reduce conflicts by setting barriers or designing special pedestrian streamline. Therefore, this paper discusses the characteristics of pedestrian flow under different weaving area setting.

First of all, we use bidimensional rectangular grids to display the motion space of pedestrian flows. We set the size of a cell to $0.4 \times 0.4 \mathrm{~m}^{2}$, which is the typical space occupied by a pedestrian. The unit of other variables is the number of cells, the overall length $L$ is 100 , and the width of a single channel $P$ is 10 . The width of bottleneck between barriers is denoted by $W$, whose value depends on different setting of weaving area. Each cell can be occupied by only one pedestrian or facility.

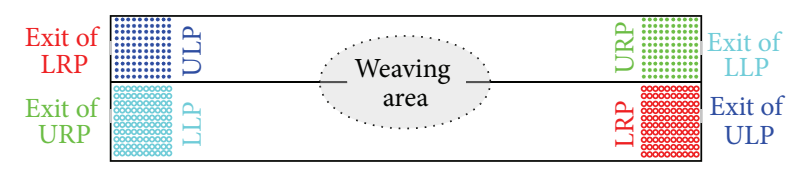

FIgURE 1: The single weaving area of pedestrian flow.

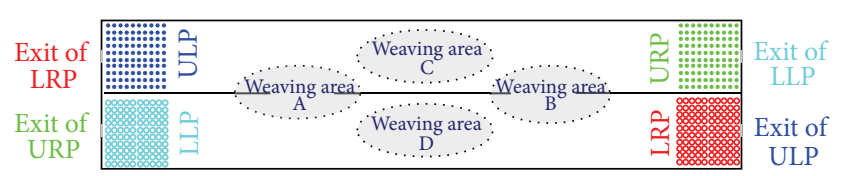

FIGURE 2: The extended weaving area of pedestrian flow.

Pedestrians are randomly distributed in four special regions on both sides at the initial time, representing four groups of pedestrians with different exits. The maximum number of pedestrians in each group is 100 . Because each group of pedestrians has an exclusive exit, thus the weaving area is formed around the bottleneck between barriers.

In this paper, the pedestrians flow scenario is formed as follows. The upper left pedestrians (ULP) go through the weaving area; they leave from the lower right exit. In the same manner, the lower left pedestrians (LLP) leave from the upper right exit, the upper right pedestrians (URP) leave from the lower left exit, and the lower right pedestrians (LRP) leave from the upper left exit. The locations of four exits are defined individually, which will be illustrated later according to different settings of weaving area. Certainly, we can consider adding the streamline of ULP leave from the lower left exit in order to form a more complex scenario, but, due to space limitation, this paper only considers the characteristics of pedestrian flow in the weaving area as above.

To exploit the weaving area effectively, the administration department sets guide signs in the weaving area to instruct pedestrians to right preference, which matches the walking habit of pedestrians. Section 3 will describe the pedestrian motion rules according to the floor field and the habit of right preference.

We mainly discuss two setting modes of the weaving area. One is the single weaving area, as shown in Figure 1, in which pedestrians of four directions weave in the central bottleneck. The other one, as shown in Figure 2, is called "extended weaving area," where the traffic administrative department should set guide signs on floor or wall, in order to let pedestrians know possible walking direction to the exits. For example, as to ULP, the guide signs should provide information that pedestrians can reach their destination by A or B, so that pedestrians can make a rational choice according to pedestrian density nearby. Under this extended setting, the weaving area can be divided into four parts: pedestrian weaving flow in region A is mainly formed by ULP and LLP; pedestrian weaving flow in region $B$ is mainly formed by URP and $\mathrm{LRP}$; region $\mathrm{C}$ and region $\mathrm{D}$ are complex regions, where pedestrians from any direction may appear in these two regions at the same time. However, most of the pedestrians from URP and LRP appear in region C and most of the pedestrians from ULP and LLP appear in region $\mathrm{D}$. 


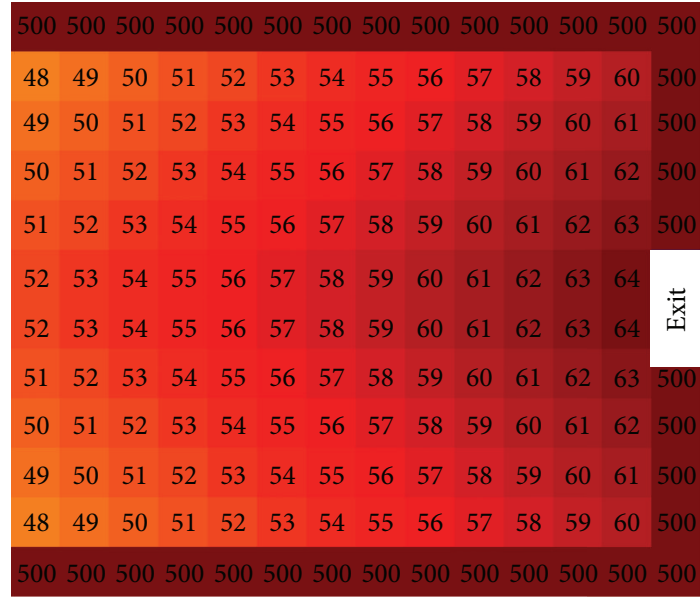

(a) $\varepsilon=1$

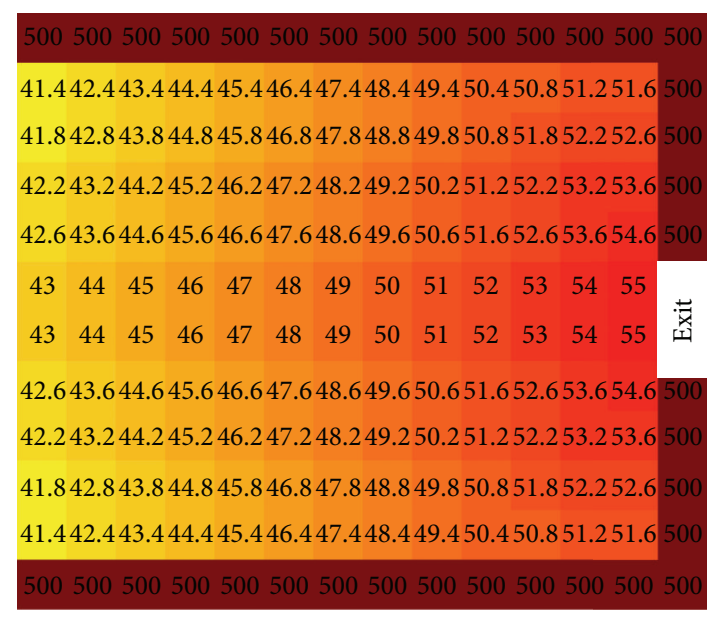

(b) $\varepsilon=0.4$

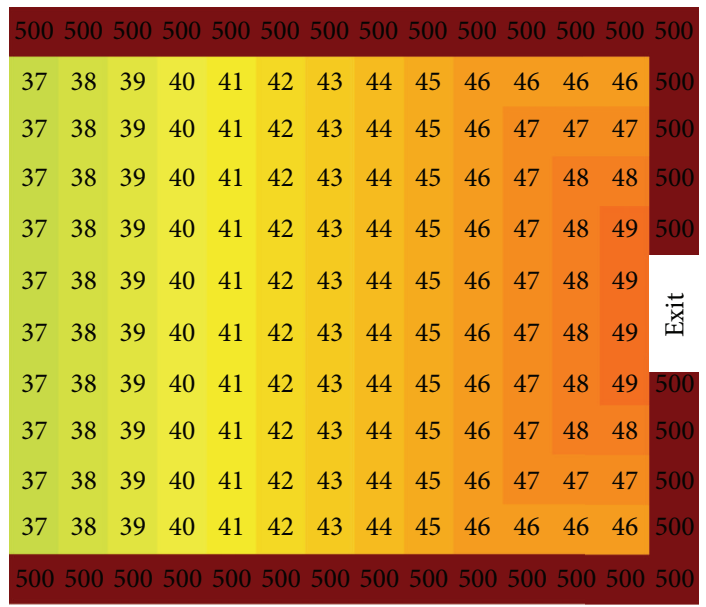

(c) $\varepsilon=0$

FIGURE 3: The static floor field formed by the exit under different values of $\varepsilon$.

The position and number of bottlenecks between barriers are related to the characteristics of pedestrian weaving flow, which will be discussed in Section 4 .

\section{The Cellular Automaton Model Based on Pedestrian Walking Habit and Floor Field}

At any time, every pedestrian follows the Moore motion rule, in which the specific transition probability calculation depends on specific floor field and the habit of right preference.

3.1. Floor Field. In the process of calculation of floor field, when pedestrians only walk in horizontal and vertical directions, the minimum step between cell $(i, j)$ and exit $m$ is denoted by $f_{i j}^{m}$. When pedestrians walk in horizontal, vertical, and diagonal directions, the minimum step between cell $(i, j)$ and exit $m$ is denoted by $e_{i j}^{m}$. The weighted distance to the exit $m$ is denoted by $d_{i j}^{m}=\varepsilon f_{i j}^{m}+(1-\varepsilon) e_{i j}^{m}$. When calculating the distance, the coefficient between $f_{i j}^{m}$ and $e_{i j}^{m}$ is denoted by $\varepsilon$. The analysis suggests that if $\varepsilon=1$, that is, pedestrians only walk in horizontal and vertical directions, a triangle would emerge near the exit (see Figure 3(a)). However, if $\varepsilon=0$, that is, pedestrians can also walk in a diagonal direction, a rectangle would emerge near the exit (see Figure 3(c)). Therefore, the value of $\varepsilon$ should depend on the arching emerging near the exit. Considering the scenarios discussed in this paper, experiment showed that if $\varepsilon=0.4$, an arched floor field would emerge (see Figure 3(b)). The value of the static floor field at cell $(i, j)$ formed by exit $m$ is denoted by $S_{i j}^{m}=$ $\max d_{i j}^{m}-d_{i j}^{m}$. When four groups of pedestrians pass the studied region, they move along the respective static floor field formed by the corresponding exit and the habit of right preference. When all pedestrians reach their exits, the simulation is terminated.

The simulation procedure of the static floor field is described as follows. 


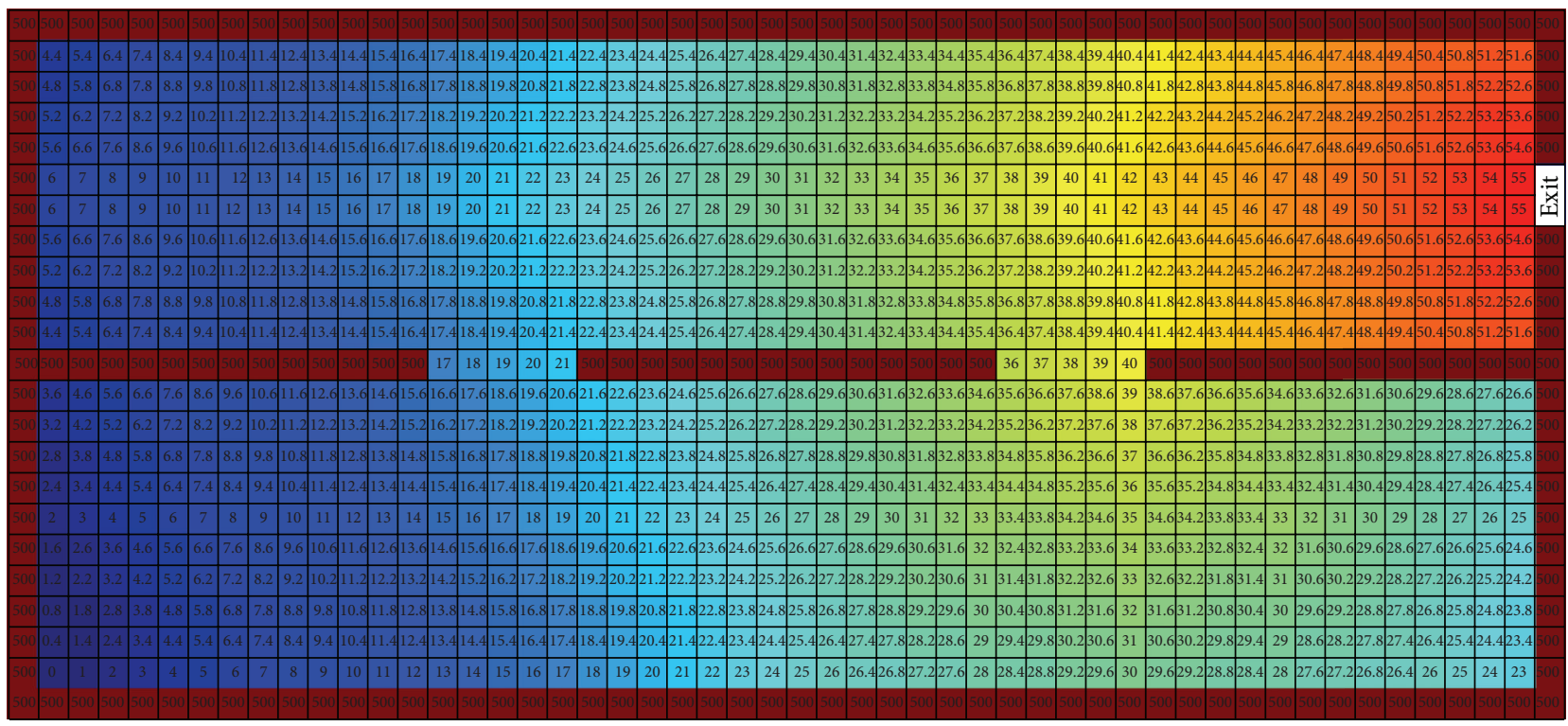

FIGURE 4: The static floor field formed by the upper right exit under a scenario with the extended weaving area.

(1) Set $k=1$, for each cell $(i, j)$, we initialize the minimum steps as follows:

$$
\begin{aligned}
f_{i j}^{m} & = \begin{cases}-1, & (i, j) \text { is wall or barrier, } \\
0, & \text { otherwise, }\end{cases} \\
e_{i j}^{m} & = \begin{cases}-1, & (i, j) \text { is wall or barrier, } \\
0, & \text { otherwise. }\end{cases}
\end{aligned}
$$

(2) Calculate the distance to the exit in four directions (horizontal and vertical):

(2.1) check grids in four directions around the exits, namely, front, rear, left, and right, denoted by $\left(i^{\prime}, j^{\prime}\right)$; if $f_{i^{\prime} j^{\prime}}^{m}=0$, then set $f_{i^{\prime} j^{\prime}}^{m}=k$;

(2.2) if $\exists i, j, f_{i j}^{m}=0$, repeat (2.2); otherwise, go to (3).

For arbitrary cell where $f_{i j}^{m}=k$, check cells in four directions around it, namely, front, rear, left, and right denoted by $\left(i^{\prime}, j^{\prime}\right)$; if $f_{i^{\prime} j^{\prime}}^{m}=0$, then set $f_{i^{\prime} j^{\prime}}^{m}=k+1$, $k=k+1$.

(3) Set $k=1$; calculate the distance to the exit in 8 directions (horizontal, vertical, and diagonal):

(3.1) check the cells around the exit in 8 directions, denoted by $\left(i^{\prime}, j^{\prime}\right)$; if $e_{i^{\prime} j^{\prime}}^{m}=0$, then set $e_{i^{\prime} j^{\prime}}^{m}=k$;

(3.2) if $\exists i, j, e_{i j}^{m}=0$, repeat (3.2); otherwise go to (4).

For arbitrary cell where $e_{i j}^{m}=k$, check $\left(i^{\prime}, j^{\prime}\right)$ around it in 8 directions; if $e_{i^{\prime} j^{\prime}}^{m}=0$, then set $e_{i^{\prime} j^{\prime}}^{m}=k+1$, $k=k+1$.

(4) For arbitrary cell $(i, j)$ in the region, calculate the weighted distance $d_{i j}^{m}=\varepsilon f_{i j}^{m}+(1-\varepsilon) e_{i j}^{m}$.
(5) For arbitrary cell $(i, j)$ in the region, calculate the static floor field value $S_{i j}^{m}=d-d_{i j}^{m}$, in which $d=$ $\max _{i, j} d_{i j}^{m}$.

Based on the simulation procedure above, Figure 4 shows the static floor field formed by the upper right exit. In this simulation, $\varepsilon$ is equal to 0.4 , the length of the pedestrian movement space is 50 , the width of each channel is 10 , and the width of each bottleneck is 5 . The number in each cell is the static floor field value of the cell, which is calculated at step (5) in the simulation procedure above. A larger number means a position closer to the exit. As can be seen in Figure 4, an arching contour is formed around the exit. This means that our parameter setting reflects the phenomenon of "arch-like blockings of the exit" [16]. We set the overall length $L$ to 50 here in order to display a clear figure in a single page, because of the large volume of numbers. In other experiments, the overall length $L$ is set as 100 . Other settings and the static floor field formed by different exits are similar to this example.

3.2. Local Motion Rules. The adopted Moore motion rule is as shown in Figure 5, and the corresponding transition probability is shown in Figure 6.

The transition probability of pedestrians walking is calculated as follows:

$$
P_{i j}^{m}=N \exp \left(k_{S} S_{i j}^{m}\right) \exp \left(k_{D} D_{i j}^{m}\right)\left(1-\mu_{i j}\right) \xi_{i j}
$$

where $N$ is the normalized factor:

$$
N=\left(\sum_{i, j} \exp \left(k_{S} S_{i j}^{m}\right) \exp \left(k_{D} D_{i j}^{m}\right)\left(1-\mu_{i j}\right) \xi_{i j}\right)^{-1}
$$




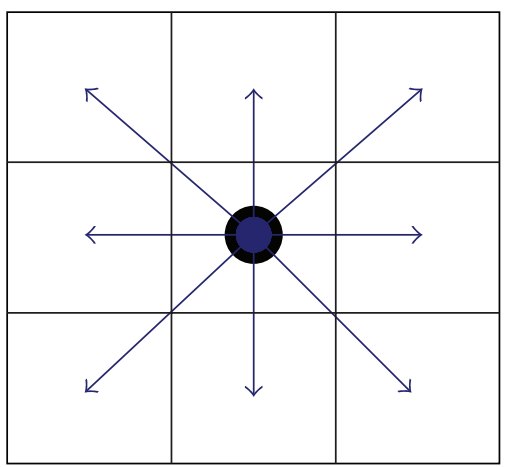

Figure 5: Moore motion rule.

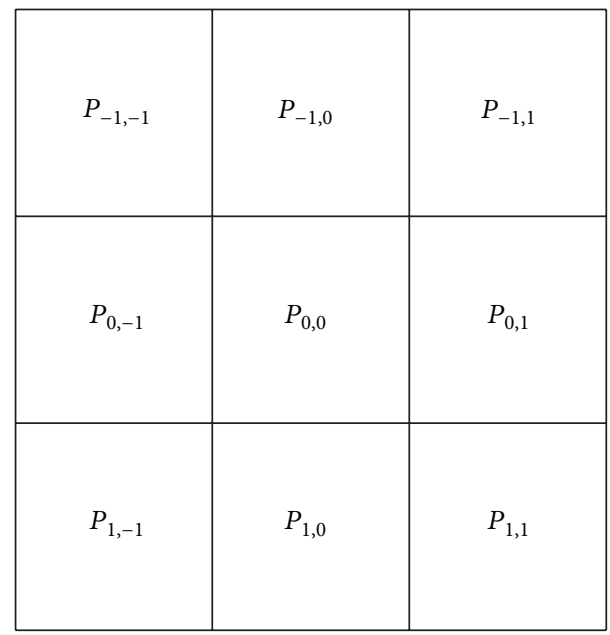

FIGURE 6: Transition probability.

$$
\begin{aligned}
& \mu_{i j}= \begin{cases}1, & (i, j) \text { is occupied, } \\
0, & \text { otherwise }\end{cases} \\
& \xi_{i j}= \begin{cases}0, & (i, j) \text { is wall or barrier, } \\
1, & \text { otherwise. }\end{cases}
\end{aligned}
$$

$S_{i j}^{m}$ is the value of the static floor field at cell $(i, j)$ formed by exit $m$, and $D_{i j}^{m}$ is the value of the dynamic floor field at cell $(i, j)$ formed by exit $m$. The coefficient of the static floor field $k_{S}$ and the coefficient of the dynamic floor field $k_{D}$ have remarkable influence on the distribution of the floor field values. $k_{S}$ reflects the degree of pedestrians' familiarity to the inherent characteristics of the scenario, whereas $k_{D}$ reflects the following trend of pedestrian in procession. Kirchner and Schadschneider [15] analyzed $k_{S}$ and $k_{D}$ in detail, and they pointed out that if $k_{D}$ is set as a fixed value, when $k_{S}$ increases gradually, the pedestrians' motion time would decrease in a nonlinear way. In other words, the more familiar the pedestrian is to the scenario, the shorter time the procession needs. Meanwhile, if $k_{S}$ is fixed, when $k_{D}$ increases gradually, the pedestrians' motion time would increase when it exceeds a certain threshold, especially when $k_{S}$ is small. This reflects that when pedestrians are not familiar with the scenario,

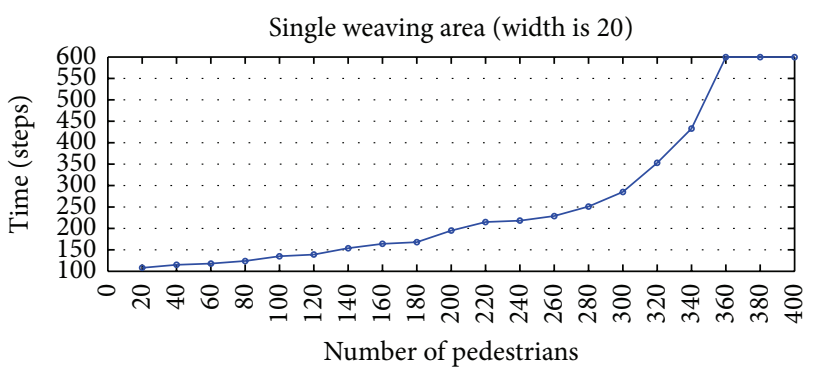

FIGURE 7: The relationship between the number of pedestrians and the time taken by using single weaving area.

following other pedestrians aimlessly would increase the motion time [15].

Since the scenario discussed in this paper is passenger terminal, we can treat pedestrians as people who have specific aim. Thereby, there is no need to consider the aimless following phenomenon in emergency, so that only the static floor field is adopted in this research. In simulation, we set the coefficient of dynamic floor field $k_{D}$ to zero.

3.3. The Strategy of Right Preference. Before entering the weaving area, pedestrians choose the next cell by transition probability based on the floor field. After a pedestrian enters the weaving area, we first calculate the cell that the pedestrian should enter based on the formula of transition probability. If the cell is occupied by another pedestrian, then pedestrian will follow the strategy of right preference and choose the offside of this cell. The "offside" here means the right side of the cell which is chosen by current pedestrian but is occupied by another pedestrian. If one vacant cell is chosen by more than one pedestrian, then we will choose one pedestrian randomly to determine who shall enter this cell. If there is no available cell, the pedestrian should stay in the cell he occupied until next iteration.

\section{The Simulation Experiment of Pedestrian Flow}

The simulation experiment is carried out in MATLAB 2012. The maximum number of iterations is 600. In Figure 7, which indicates the relationship between the number of pedestrians and the time taken (steps), if the time taken equals 600, it means that the clogging appears in simulation.

4.1. Simulation of Pedestrian Weaving Flow by Using Single Weaving Area Setting. If one adopts single weaving area, it is easy to cause clogging when pedestrians in four directions approach the weaving area at the same time. Figure 7 shows the relationship between the number of pedestrians and the time taken by using single weaving area. In this simulation, the total width of the bottleneck is set as 20 . The average time taken for each number of pedestrians is based on 50 independent experiments. Figure 7 shows that the time taken under single weaving area increases sharply when the number of pedestrians reaches more than 200. Such time increase is 


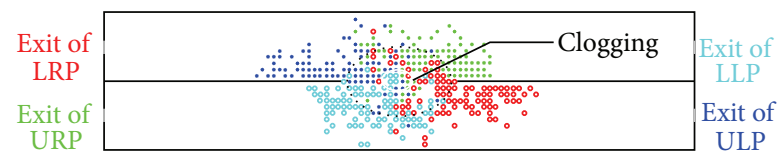

FIGURE 8: Clogging phenomenon in the single weaving area.

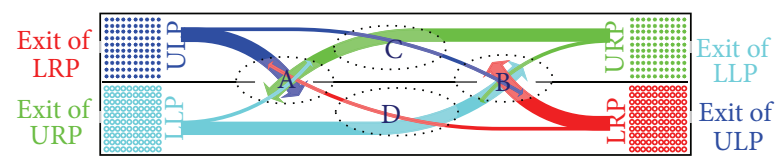

FIgURE 9: Streamline net of pedestrian weaving flow.

caused by detour, layering, and clogging of the pedestrians. Furthermore, as can be seen in Figure 8, when the number of pedestrians reaches more than 360, a clear clogging phenomenon can be observed in the weaving area. We should also notice in Figure 7 that there are still some pedestrians who cannot leave the region and even the number of iterations reaches 600 .

\subsection{Simulation of Pedestrian Weaving Flow by Using Extended} Weaving Area Setting. By setting the extended weaving area as shown in Figure 2, the weave of streamline becomes more discrete in time and space and thus could avoid congestion effectively. Figure 9 shows the streamline net of pedestrian weaving flow by using extended weaving area setting. We use different line width to represent different densities of pedestrians. As can be seen from Figures 9 and 10, most of pedestrians walk in right direction, which means the habit of right preference is reflected in our model.

To observe how the extended weaving area performs, in this subsection, we set the width of every bottleneck as 10 (the total width is 20) and the total number of pedestrians as 200 (50 people in each group) as an example to show every stage of pedestrian weaving. As shown in Figures 9 and 10, the position of two bottlenecks is set at the proper location, so as to divide the central isolation barrier into three appropriately equal parts. The separation between the two bottlenecks is 26 , and the other parts on both sides are 27, respectively.

Figure 10(a) shows the scenario at the initial time (step 0), in which the four groups of pedestrians are distributed randomly in the initial region. Figure 10(b) shows the scenario at step 31, the first stage of pedestrians' weaving march. Weaving of ULP and LLP takes place in region A, and weaving of URP and LRP takes place in region B. In order to avoid four groups of pedestrians entering the same weaving area at the same time, both A and B only have two groups of pedestrians who take part in different stage; as can be seen from Figures 9 and 10 , most of pedestrians walk in right direction, which means that the habit of right preference is reflected by our model. In our simulation, pedestrians will clog around bottlenecks as shown in Figure 10 if right preference is not involved in the simulation. Figure 10(c) shows the scenario at step 58, the second stage of pedestrians' weaving march; pedestrians weaving take part in $\mathrm{C}$ and $\mathrm{D}$ separately. In region $\mathrm{C}$ and region $\mathrm{D}$, all of the four groups of pedestrians have shown up.

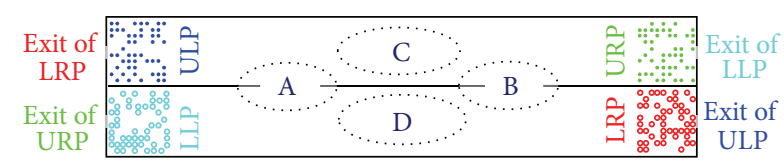

(a) Initial time

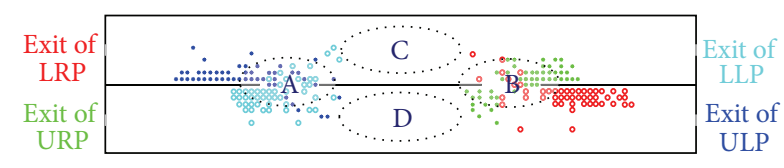

(b) The first stage of pedestrian weaving flow

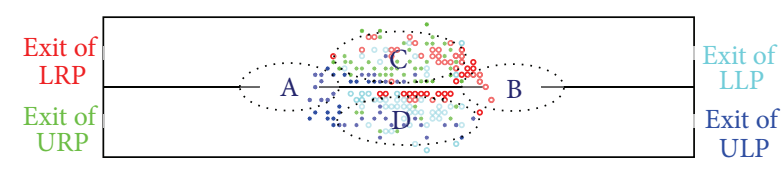

(c) The second stage of pedestrian weaving flow

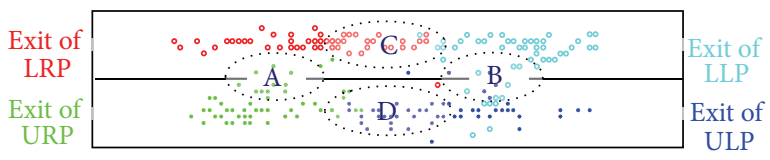

(d) The stage of pedestrians leaving weaving area

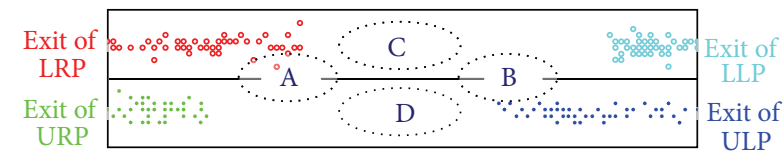

(e) Pedestrians arrive at their respective exits

FIGURE 10: Each stage during the pedestrians weaving by using the extended weaving area. (a) Initial time; (b) the first stage of pedestrian weaving flow; (c) the second stage of pedestrian weaving flow; (d) the stage of pedestrians leaving weaving area; (e) pedestrians arrive at their exits.

However, due to distributary in the first stage, in region $\mathrm{C}$ it appears that most URP and LRP walk in left direction and a few ULP and LLP walk in right direction. In region D, it appears that most ULP and LLP walk in right direction and a few URP and LRP walk in left direction. The reason why LLP show in region $D$ is when LLP walk across $A$, there will be collisions because of the weaving of ULP and LLP, so as for LLP, the front region (region B) still has access to the upper right exit. In this way, part of LLP do not enter region $A$ but instead enter region $\mathrm{B}$ through region $\mathrm{D}$ and then walk to their exit. We can explain the appearance in $\mathrm{C}$ and $\mathrm{D}$ of the other three types of pedestrians in a similar way. Figure 10(d) shows the scenario at step 89; when pedestrians leave the weaving area, they walk to their respective exits. Figure 10(e) shows the scenario at step 119; pedestrians arrive at the respective exits one after another.

From the detailed simulation process, we can see that, with the extended weaving area setting, the weaving of pedestrians from four directions is divided into two stages, respectively, in two kinds of area. The experiment shows that by setting of extended weaving area, the weaving is discretized in time and space, and the clogging and conflicts are avoided effectively. In particular, as is shown above, according to 


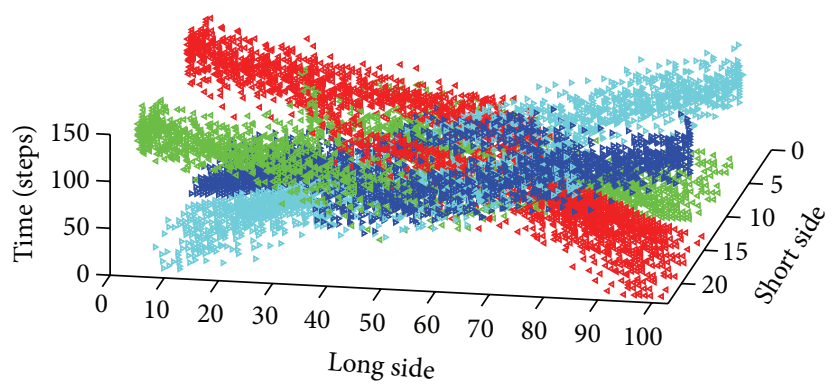

FIGURE 11: The spatial and temporal distribution of the pedestrian flow with the extended weaving area.

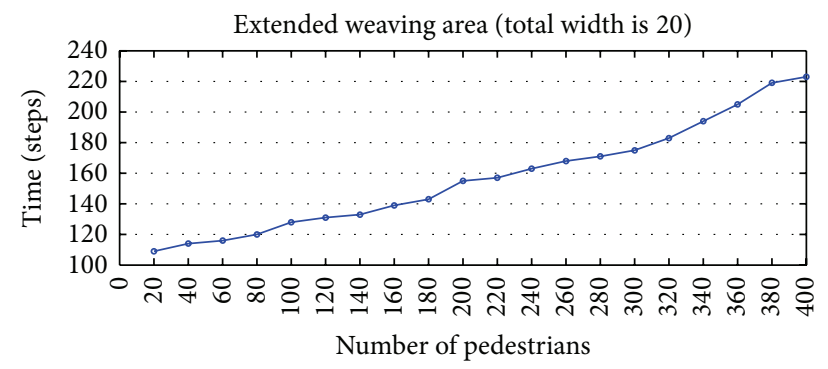

FIGURE 12: The relationship between the number of pedestrians and the time taken by using the extended weaving area.

the information offered by the traffic administration, part of pedestrians can choose not to enter in the bottleneck when weaving first appears and keep going forward to the bottleneck ahead. However, when these pedestrians enter the bottleneck, there mainly only has this part of pedestrians, or other pedestrians are very rare (see the left bottleneck as shown in Figure 10(d)). In this way, clogging in weaving area can be relieved. But in single weaving area, pedestrians only have one choice; thus it is easy to cause clogging when large numbers of pedestrians reach the threshold.

Figure 11 shows the spatial and temporal distribution of pedestrians shown in situations in Figure 10. The $z$-axis denotes time (steps), the $x$-axis denotes the long side of the research region, and the $y$-axis denotes the short side of the research region. In Figure 11, different colors of the triangles represent different groups of pedestrians. As can be seen in Figure 11, at the initial stage (corresponding to Figure 10(a)) and at the final stage (corresponding to Figure 10(e)), there is no weaving, and the pedestrians walk towards their exits, respectively. The most weaving happens when pedestrians enter the weaving area (corresponding to Figures 10(b)$10(\mathrm{~d})$ ), where we can observe the pedestrians overlap with each other severely.

\subsection{Comparison between the Single Weaving and the Extended} Weaving Area. The relationship between the number of pedestrians and the time taken by using the extended weaving area is shown in Figure 12. In this simulation, the total width of two bottlenecks is set as 20 . The average time taken for each number of pedestrians is also based on 50 independent experiments. In addition, as can be seen in Figure 12, the

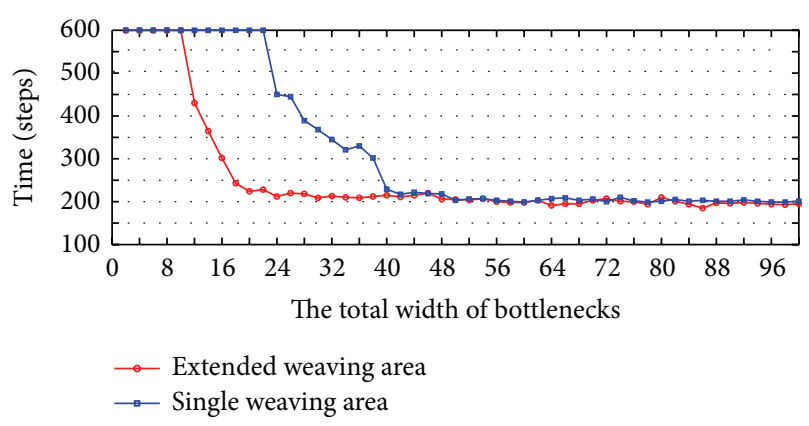

FIGURE 13: The relationship between the width of bottleneck and the time taken.

time taken by using extended weaving area is significantly less than the time taken by using signal weaving area (in Figure 7). As aforementioned, by using extended weaving area, special streamline of pedestrian flow can be formed to avoid unnecessary collisions, and it can also bring convenience for administration.

The relationship between the time taken and the total width of bottlenecks is shown in Figure 13. In this simulation, the number of pedestrians is 400 , and the average time taken for each number of the total width of bottlenecks is based on 50 independent experiments. When adopting the extended weaving area, if the width of single bottleneck is less than 6 (the total width of two bottlenecks is less than 12), there will be a long-time clogging and some pedestrians who walk behind others will walk around randomly. When the width of single bottleneck is 7-10 (the total width of two bottlenecks is 1420 ), the time will decrease gradually, and transitory clogging will occur. In pedestrians' gathering time, we can notice the phenomena of detour and layering. From the discussion above, we can draw the following conclusion: in order to avoid clogging, the width threshold of single bottleneck in the extended weaving area is 6; that is, the total width threshold of bottlenecks in the extended weaving area is 12 .

From Figure 13, we can see that when adopting the single weaving area and when the number of pedestrians is 400 , in order to avoid clogging, the width threshold of bottleneck in the single weaving area is 24 . With the same amount of pedestrians, the time taken of the single weaving area is larger than that of the extended weaving area when total width of bottlenecks is less than 40 .

As can be seen in Figure 14, by using the threshold width, the relationship between the number of pedestrians and the time taken is similar under the situation of using the single weaving area and the extended weaving area. However, the total width of bottlenecks in the extended weaving area is less than that of the single weaving area. Therefore, the extended weaving area is more effective than the single weaving area.

4.4. The Effect of the Separation between Two Bottlenecks. We would like to emphasize that the results presented in this subsection are based on 50 independent experiments. The total width of bottlenecks is set as 20 . For extended weaving area, in order to analyze the effect of the separation between 


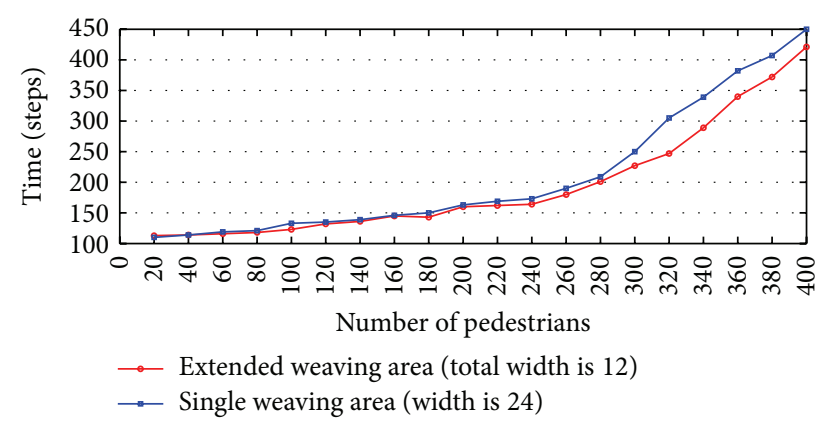

FIGURE 14: The relationship between the number of pedestrians and the time taken by using threshold width.

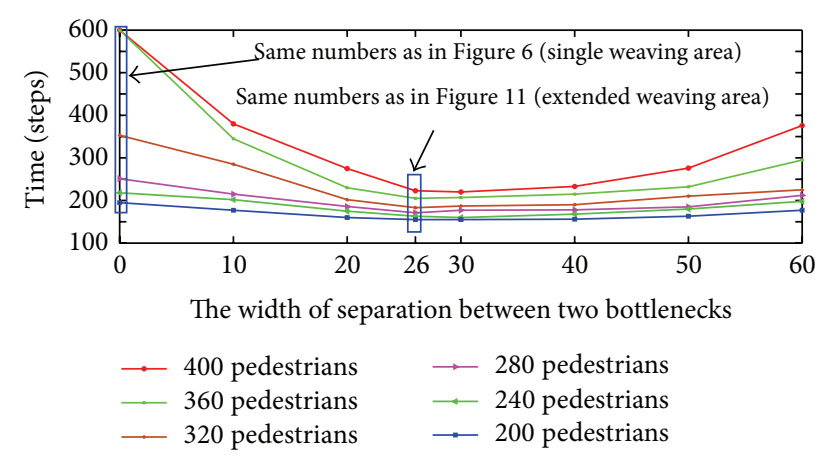

Figure 15: The effect of the separation between two bottlenecks (total width is 20).
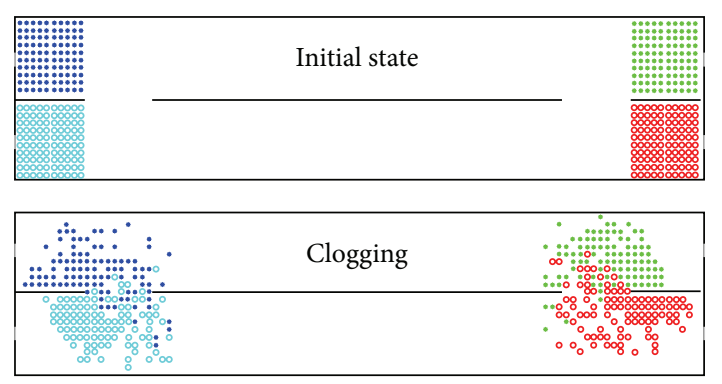

FIGURE 16: Clogging prematurely (the width of separation is 60).

two bottlenecks, we plot the relationship between the time taken and the separation between two bottlenecks under different number of pedestrians in Figure 15.

As can be seen in Figure 15, if the width of the separation is equal to zero, as in the single weaving area, the time taken is the same as that in Figure 7. Moreover, if the width of the separation is equal to 26 , as in the extended weaving area, the time taken is the same as that in Figure 12. When the width of separation is from 20 to 40 , the time taken has relatively small changes for given number of pedestrians, especially for a lesser number of pedestrians. The time taken becomes larger when the width of separation is 60 . Figure 16 illustrates the reason. We can observe four different groups of pedestrians near two bottlenecks at initial state. The pedestrians are clogging prematurely around bottlenecks, so that they cannot use the extended weaving area effectively which is shown in
Figures 2 and 9 . Therefore, the width of separation should be set between 20 and 40 for the simulation scenario in order to give full play to the extended weaving area. The role of separation is just like a column placed in front of the exits [16]. It can subdivide the crowd and the pressure and then relieve the conflicts and clogging caused by pedestrian weaving.

\section{Conclusions}

In large passenger terminal and rail transit hub, complex streamline net formed by pedestrian flows could result in emerging pedestrian weaving. The administration should adopt effective coordinated control methods to avoid the clogging caused by such pedestrian weaving in rush hours. We have proposed the concept of the extended weaving area and simulated and analyzed the dynamic characteristics of the streamline weaving area using cellular automaton model based on the floor field and human walking habits (i.e., right preference). The simulation experiment has demonstrated that when the extended weaving area is adopted and effective guide signs are given (e.g., point out the direction at appropriate location or ground), we could discretize the streamline of pedestrians in both time and space. Through simulation experiment, we have given the threshold of bottleneck in the extended weaving area in specific scenario. This setting mode and simulation method of the extended weaving area can be applied to existing environment where the widths of channel and bottleneck are limited. Meanwhile, it can also be applied to the analysis of pedestrian weaving streamline during the design period of large passenger terminal and rail transit hub.

\section{Conflict of Interests}

The authors declare that there is no conflict of interests regarding the publication of this paper.

\section{Acknowledgments}

The research is supported by the National Natural Science Foundation of China (Grant nos. 51278221 and 51378076) and the China Scholarship Council.

\section{References}

[1] D. Helbing, "Traffic and related self-driven many-particle systems," Reviews of Modern Physics, vol. 73, no. 4, pp. 1067-1141, 2001.

[2] G. Ren, L. L. Li, and W. Wang, "Modeling bi-direction pedestrian flow by cellular automata and complex network theories," Acta Physica Sinica, vol. 61, no. 14, Article ID 144501, 2012.

[3] L. L. Lu, G. Ren, W. Wang, C. Yu, and C. Z. Ding, "Exploring the effects of different walking strategies on bi-directional pedestrian flow," Discrete Dynamics in Nature and Society, vol. 2013, Article ID 150513, 9 pages, 2013.

[4] Y. Zheng, B. Jia, X. G. Li, and N. Zhu, "Evacuation dynamics with fire spreading based on cellular automaton," Physica A: Statistical Mechanics and its Applications, vol. 390, no. 18-19, pp. 3147-3156, 2011. 
[5] H. Yue, C.-F. Shao, H.-Z. Guan, and L.-M. Duan, "Simulation of pedestrian evacuation flow with affected visual field using cellular automata," Acta Physica Sinica, vol. 59, no. 7, pp. 44994507, 2010.

[6] A. Kirchner, H. Klüpfel, K. Nishinari, A. Schadschneider, and M. Schreckenberg, "Simulation of competitive egress behavior: comparison with aircraft evacuation data," Physica A: Statistical Mechanics and Its Applications, vol. 324, no. 3-4, pp. 689-697, 2003.

[7] C.-K. Chen, J. Li, and D. Zhang, "Study on evacuation behaviors at a T-shaped intersection by a force-driving cellular automata model," Physica A: Statistical Mechanics and its Applications, vol. 391, no. 7, pp. 2408-2420, 2012.

[8] A. Varas, M. D. Cornejo, D. Mainemer et al., "Cellular automaton model for evacuation process with obstacles," Physica A: Statistical Mechanics and its Applications, vol. 382, no. 2, pp. 631642, 2007.

[9] E. A. Lim and W. C. Tan, "Simulating evacuations with obstacles using a modified dynamic cellular automata model," Journal of Applied Mathematics, vol. 2012, Article ID 765270, 17 pages, 2012.

[10] X. X. Jian and X. Zhang, "Potential field cellular automata model for pedestrian evacuation in a domain with a ramp," Mathematical Problems in Engineering, vol. 2014, Article ID 714267, 7 pages, 2014.

[11] C. Burstedde, K. Klauck, A. Schadschneider, and J. Zittartz, "Simulation of pedestrian dynamics using a two-dimensional cellular automaton," Physica A: Statistical Mechanics and its Applications, vol. 295, no. 3-4, pp. 507-525, 2001.

[12] H.-J. Huang and R.-Y. Guo, "Static floor field and exit choice for pedestrian evacuation in rooms with internal obstacles and multiple exits," Physical Review E: Statistical, Nonlinear, and Soft Matter Physics, vol. 78, no. 2, Article ID 021131, 2008.

[13] R. Chen, X. Li, and L. Y. Dong, "Modeling and simulation of weaving pedestrian flow in subway stations," Acta Physica Sinica, vol. 61, no. 14, Article ID 144502, 2012.

[14] L. Yao, L. Sun, Z. Zhang, S. Wang, and J. Rong, "Research on the behavior characteristics of pedestrian crowd weaving flow in transport terminal," Mathematical Problems in Engineering, vol. 2012, Article ID 264295, 9 pages, 2012.

[15] A. Kirchner and A. Schadschneider, "Simulation of evacuation processes using a bionics-inspired cellular automaton model for pedestrian dynamics," Physica A: Statistical Mechanics and Its Applications, vol. 312, no. 1-2, pp. 260-276, 2002.

[16] D. Helbing, I. Farkas, P. Molnar, and T. Vicsek, "Simulation of pedestrian crowds in normal and evacuation situations," in Pedestrian and Evacuation Dynamics, M. Schreckenberg and S. D. Sharma, Eds., Springer, Berlin, Germay, 2001.

[17] D. Helbing, I. Farkas, and T. Vicsek, "Simulating dynamical features of escape panic," Nature, vol. 407, no. 6803, pp. 487490, 2000.

[18] M. Isobe, D. Helbing, and T. Nagatani, "Experiment, theory, and simulation of the evacuation of a room without visibility," Physical Review E, vol. 69, no. 6, Article ID 066132, 2004.

[19] D. Amadori and M. di Francesco, "The one-dimensional Hughes model for pedestrian flow: riemann-type solutions," Acta Mathematica Scientia, Series B, vol. 32, no. 1, pp. 259-280, 2012.

[20] S. P. Hoogendoorn and W. Daamen, "Pedestrian behavior at bottlenecks," Transportation Science, vol. 39, no. 2, pp. 147-159, 2005.
[21] T. Kretz, A. Grünebohm, M. Kaufman, F. Mazur, and M. Schreckenberg, "Experimental study of pedestrian counterflow in a corridor," Journal of Statistical Mechanics: Theory and Experiment, no. 10, Article ID P10001, 2006.

[22] J. Zhang, W. Klingsch, A. Schadschneider, and A. Seyfried, "Ordering in bidirectional pedestrian flows and its influence on the fundamental diagram," Journal of Statistical Mechanics: Theory and Experiment, vol. 2012, no. 2, Article ID P02002, 2012.

[23] R. Nishi, H. Miki, A. Tomoeda, and K. Nishinari, "Achievement of alternative configurations of vehicles on multiple lanes," Physical Review E, vol. 79, no. 6, Article ID 066119, 2009.

[24] X. G. Li, Z. Y. Gao, and B. Jia, "Capacity analysis of type-a weaving section by using cellular automata model," in Proceedings of the 7th International Conference on Traffic and Transportation Studies (ICTTS '10), pp. 1010-1018, August 2010.

[25] L. Lei, L.-Y. Dong, T. Song, and S.-Q. Dai, "Study on the traffic flow of weaving section in elevated road system with cellular automaton model," Acta Physica Sinica, vol. 55, no. 4, pp. 17111717, 2006. 


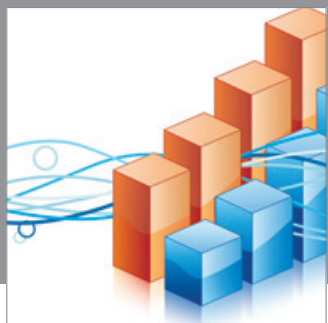

Advances in

Operations Research

mansans

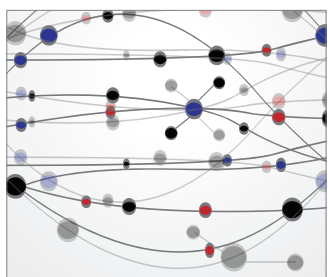

The Scientific World Journal
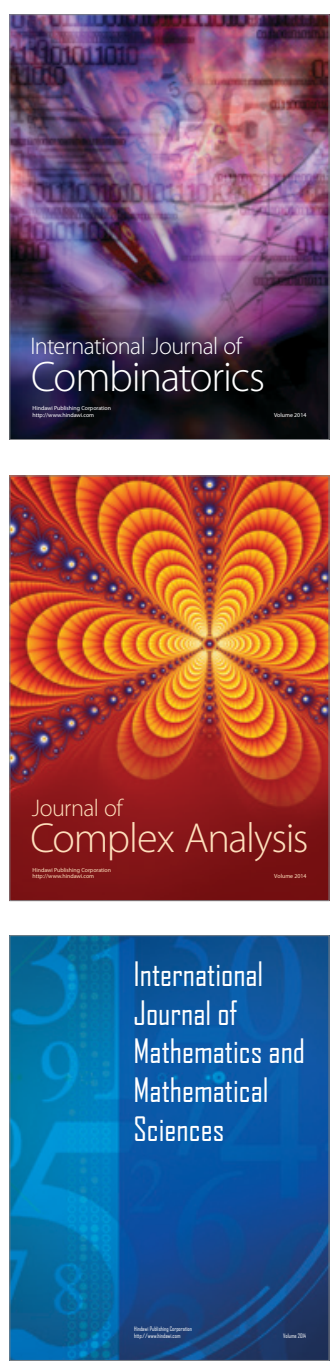
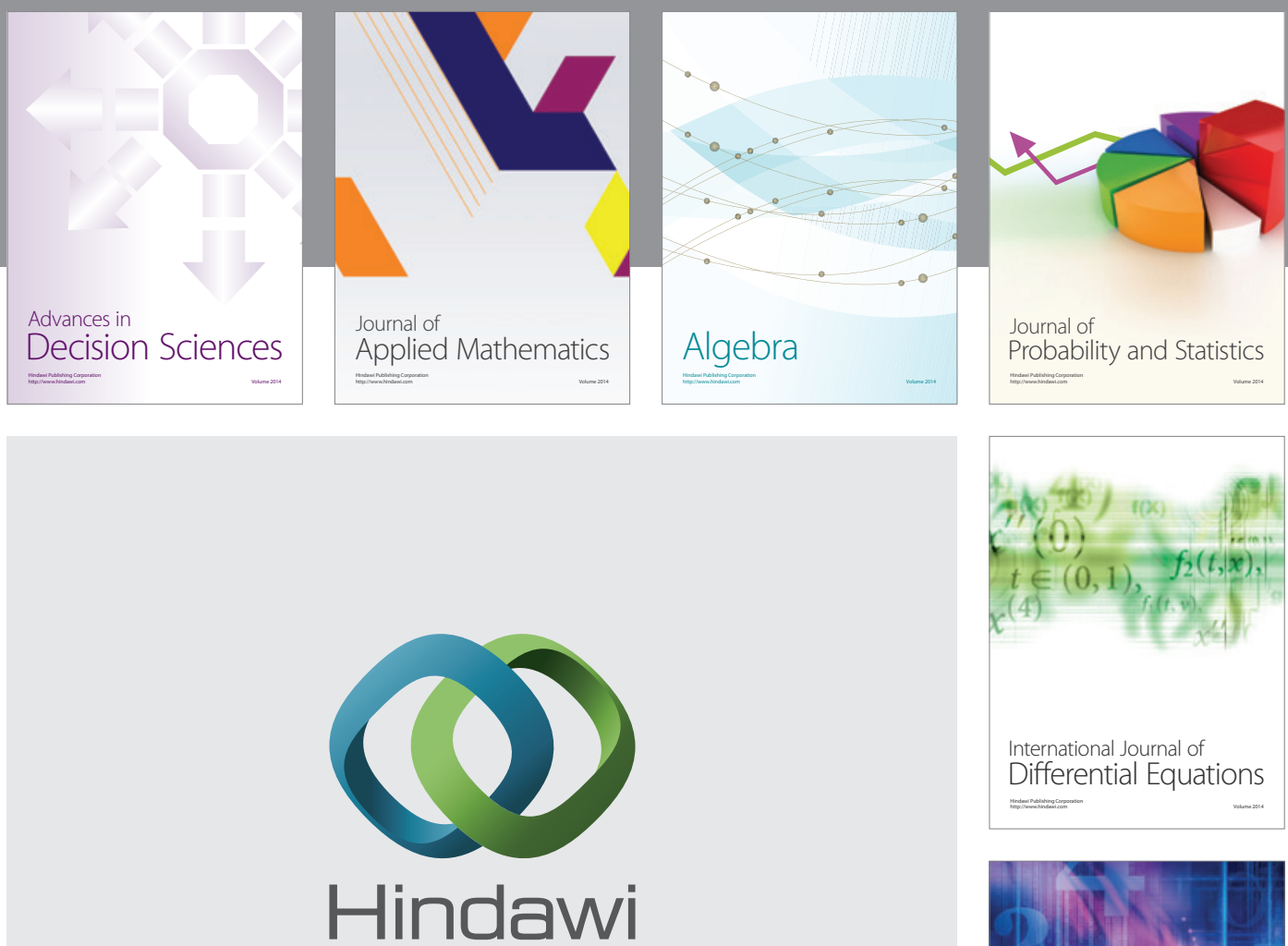

Submit your manuscripts at http://www.hindawi.com
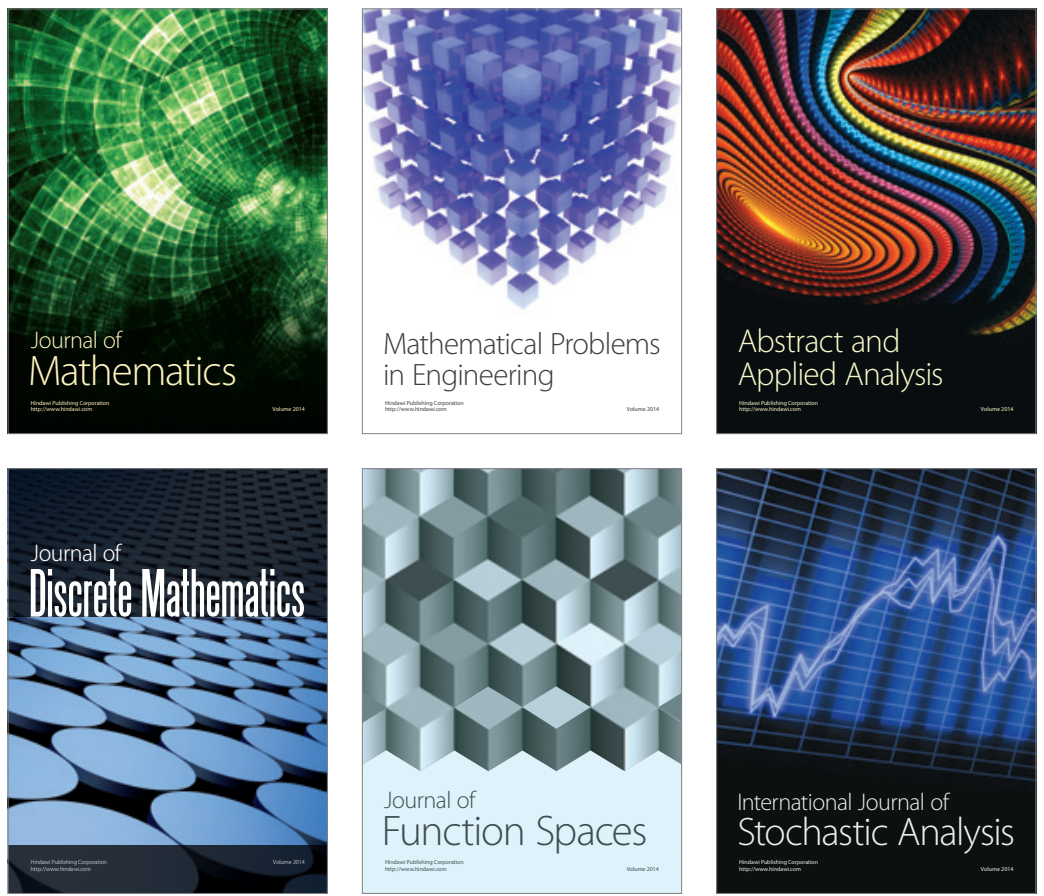

Journal of

Function Spaces

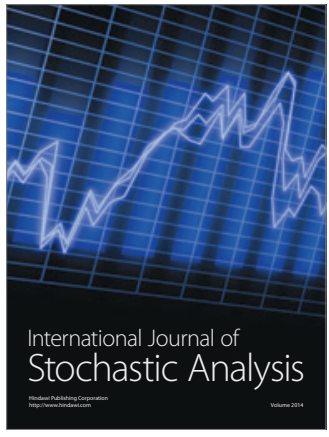

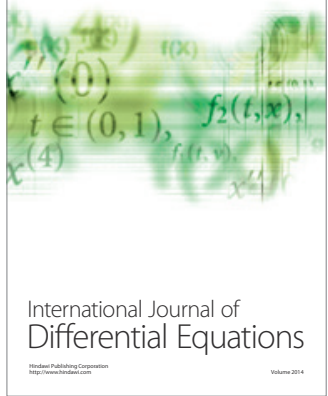
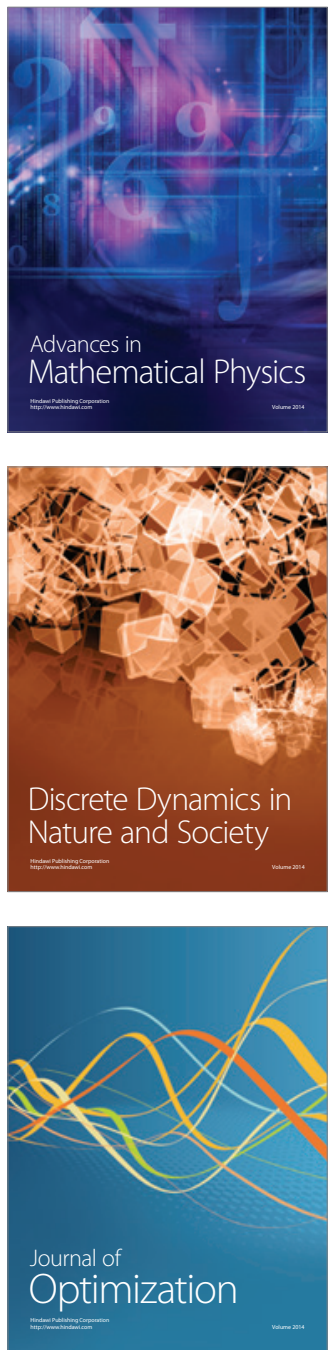